Supporting Information for

\title{
Analysis of Stereochemical Stability of Dynamic Chiral Molecules Using an Automated Microflow Measurement System
}

Kazunobu Igawa,* Shusaku Asano, ${ }^{*}$ Yuki Yoshida, Yuuya Kawasaki, and Katsuhiko Tomooka*

Institute for Materials Chemistry and Engineering, IRCCS, and Department of Molecular and Material

Sciences, Kyushu University, 6-1 Kasuga-koen, Kasuga 816-8580, Japan

*E-mail: kigawa@cm.kyushu-u.ac.jp

shusaku_asano@cm.kyushu-u.ac.jp

ktomooka@cm.kyushu-u.ac.jp

Table of Contents:

1. Analytical Data for Stereochemical Stability of DYCMs $\quad$ S2

2. Synthetic Scheme for Compound 2a

S3

3. ${ }^{1} \mathrm{H}$, and ${ }^{13} \mathrm{C}$ NMR Spectra $\quad$ S4-S9 


\section{Analytical data for stereochemical stability of DYCMs}

The enantiomeric purity of samples were determined by HPLC analysis, which were performed on a JASCO CD-2095 and MD-2018 detectors equipped with a JASCO PU-2089 using Daicel CHIRALPAK IE column $(0.46 \mathrm{~cm} \times 25 \mathrm{~cm})$ for $1 \mathrm{a}$, and IG column $(0.46 \mathrm{~cm} \times 25 \mathrm{~cm})$ for $2 \mathbf{a}$. The plot for the firstorder kinetics provided $k^{\prime}\left(=2 k_{\text {rac }}\right)$ in second.

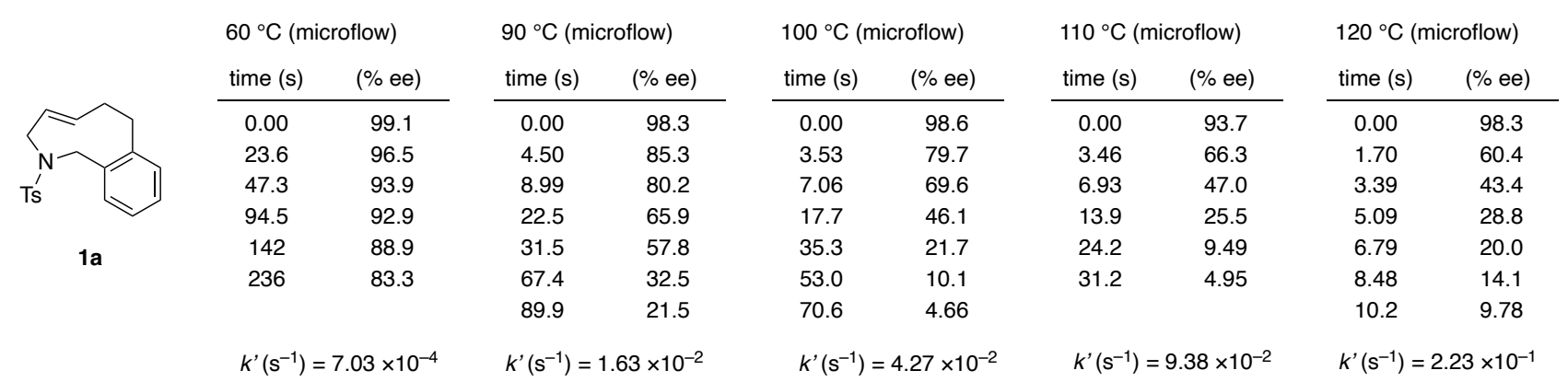

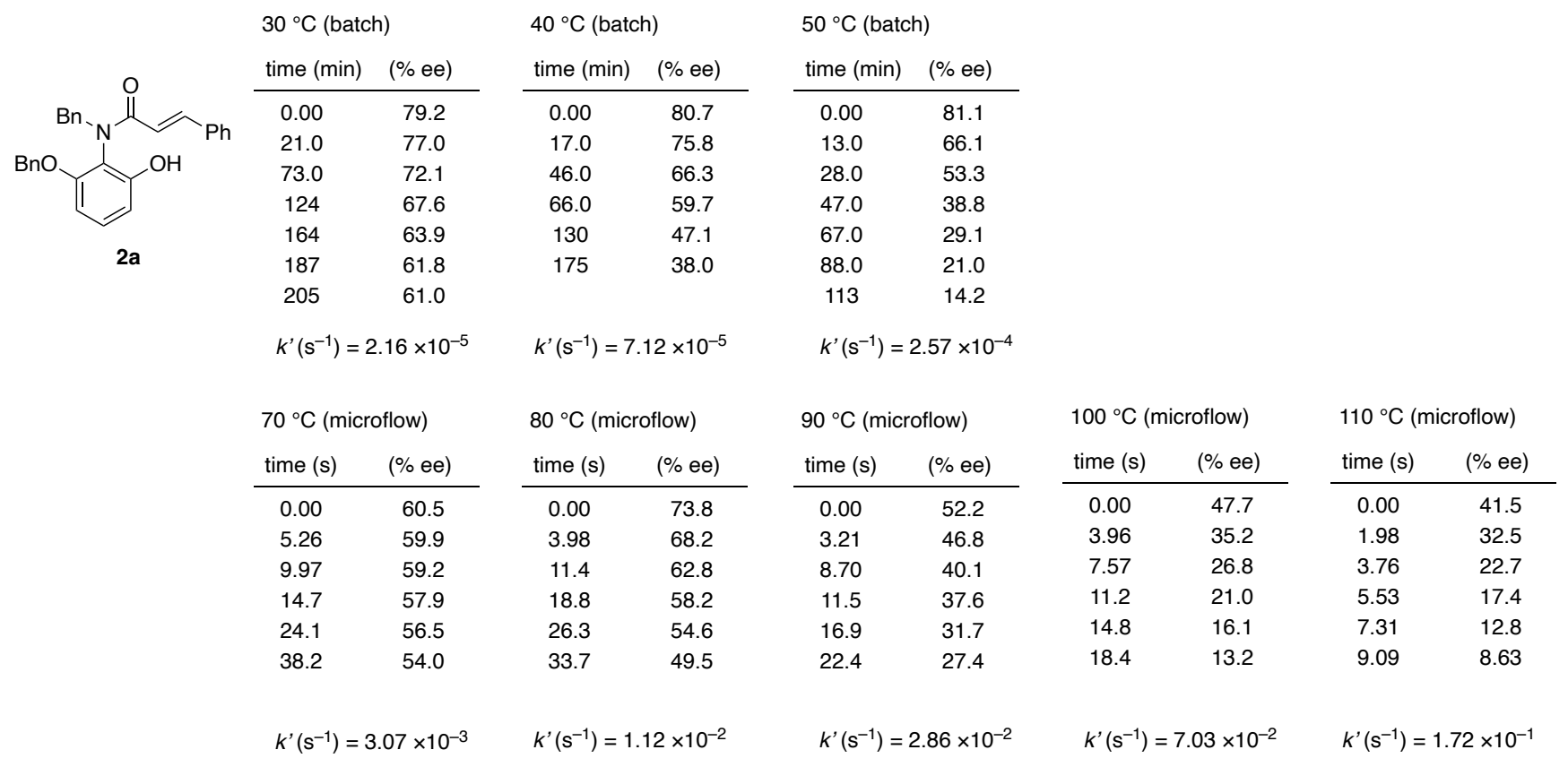




\section{Synthetic Scheme for Compound 2a}
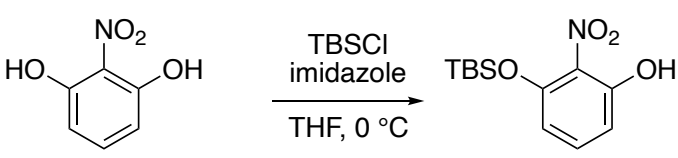

3, $70 \%$

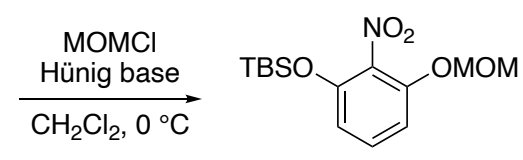

4, $76 \%$

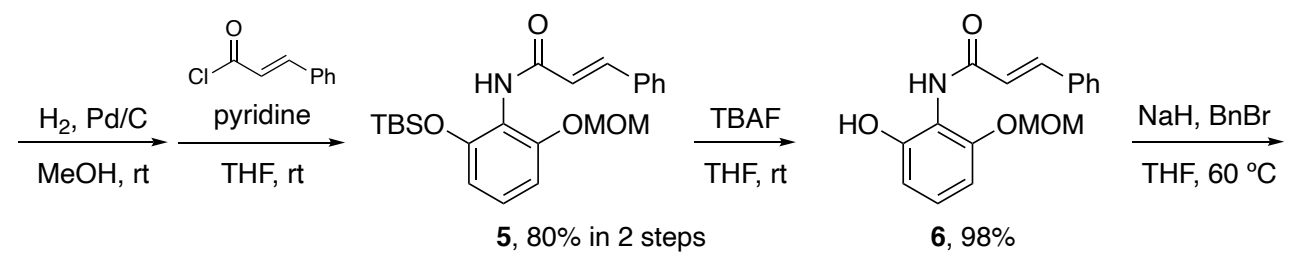

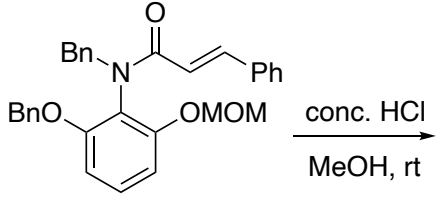

7, $94 \%$

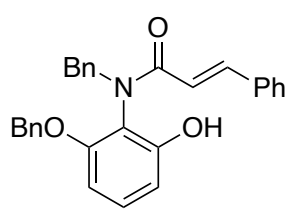

2a, 94\% 


\section{3. ${ }^{1} \mathrm{H},{ }^{13} \mathrm{C}$ NMR Spectra}

${ }^{1} \mathrm{H}$ NMR Spectrum of $\mathbf{3}\left(300 \mathrm{MHz}, \mathrm{CDCl}_{3}\right)$

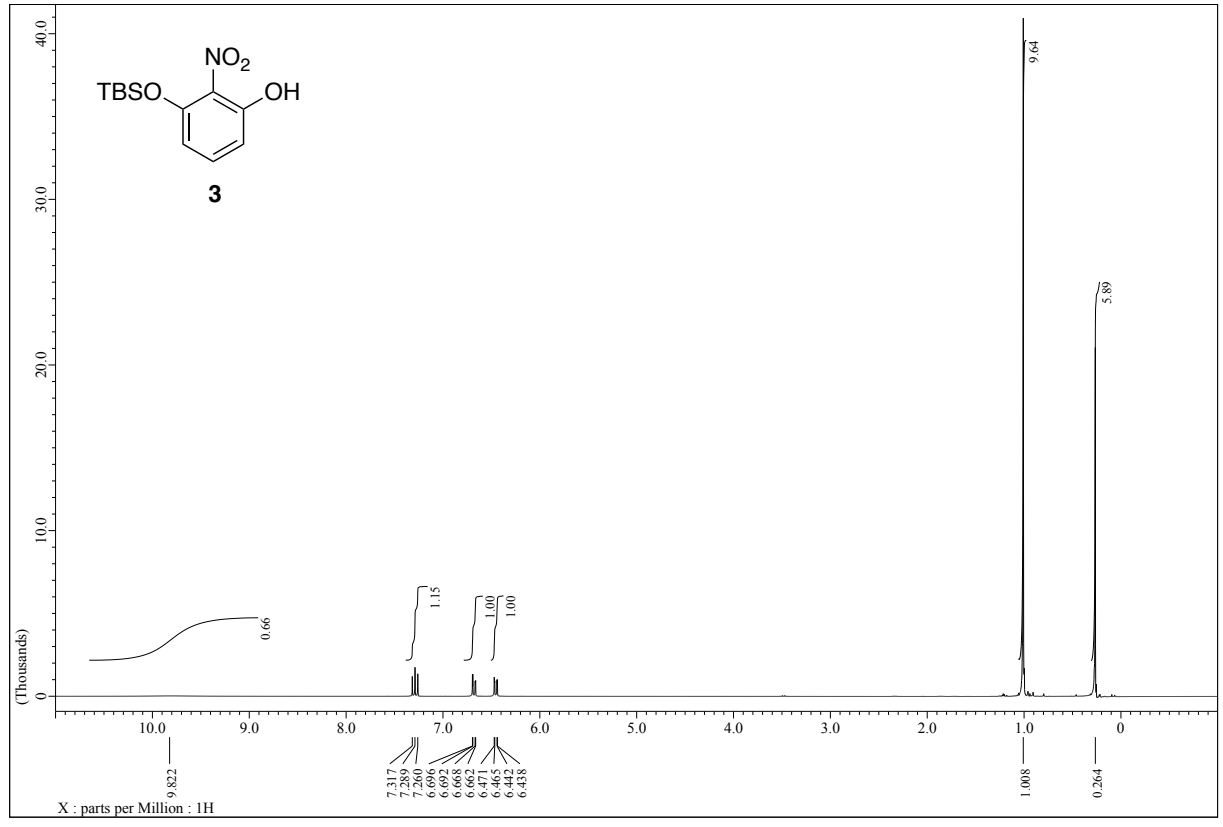

${ }^{13} \mathrm{C}$ NMR Spectrum of $\mathbf{3}\left(75 \mathrm{MHz}, \mathrm{CDCl}_{3}\right)$

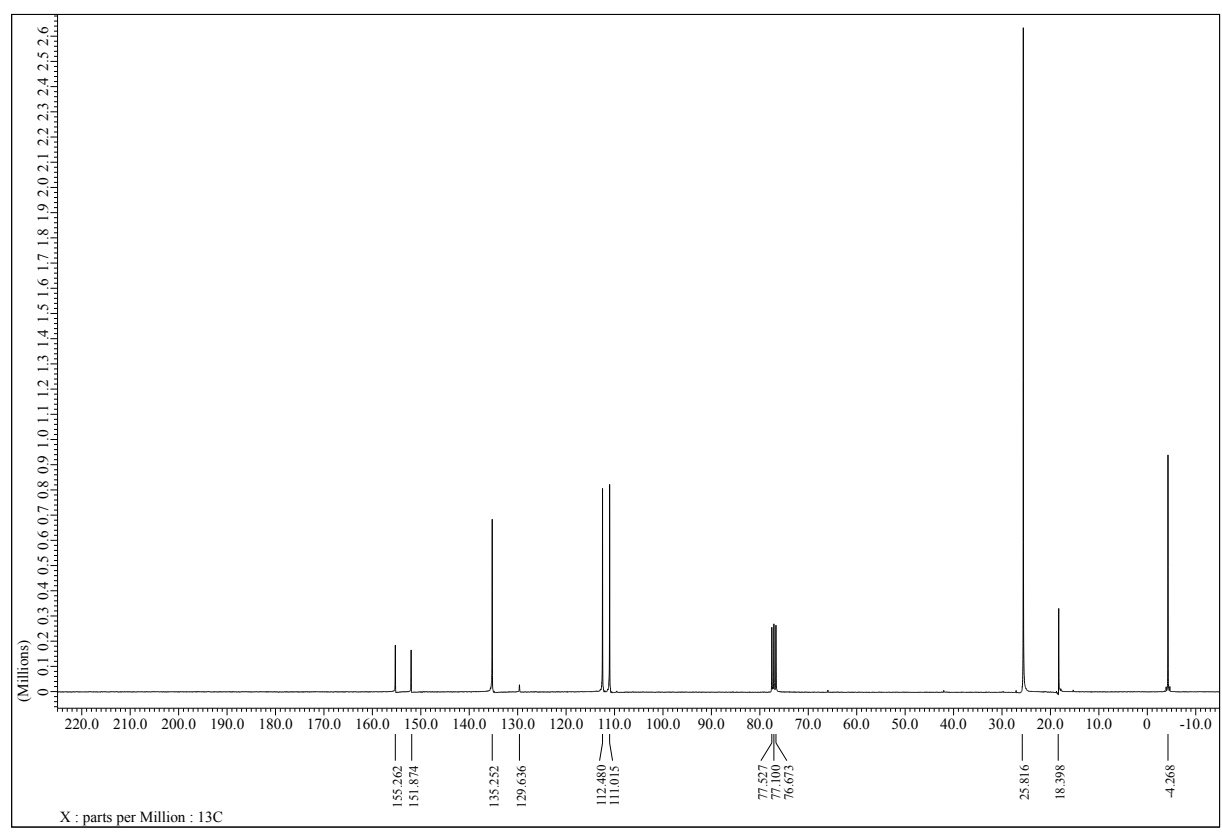


${ }^{1} \mathrm{H}$ NMR Spectrum of $4\left(300 \mathrm{MHz}, \mathrm{CDCl}_{3}\right)$

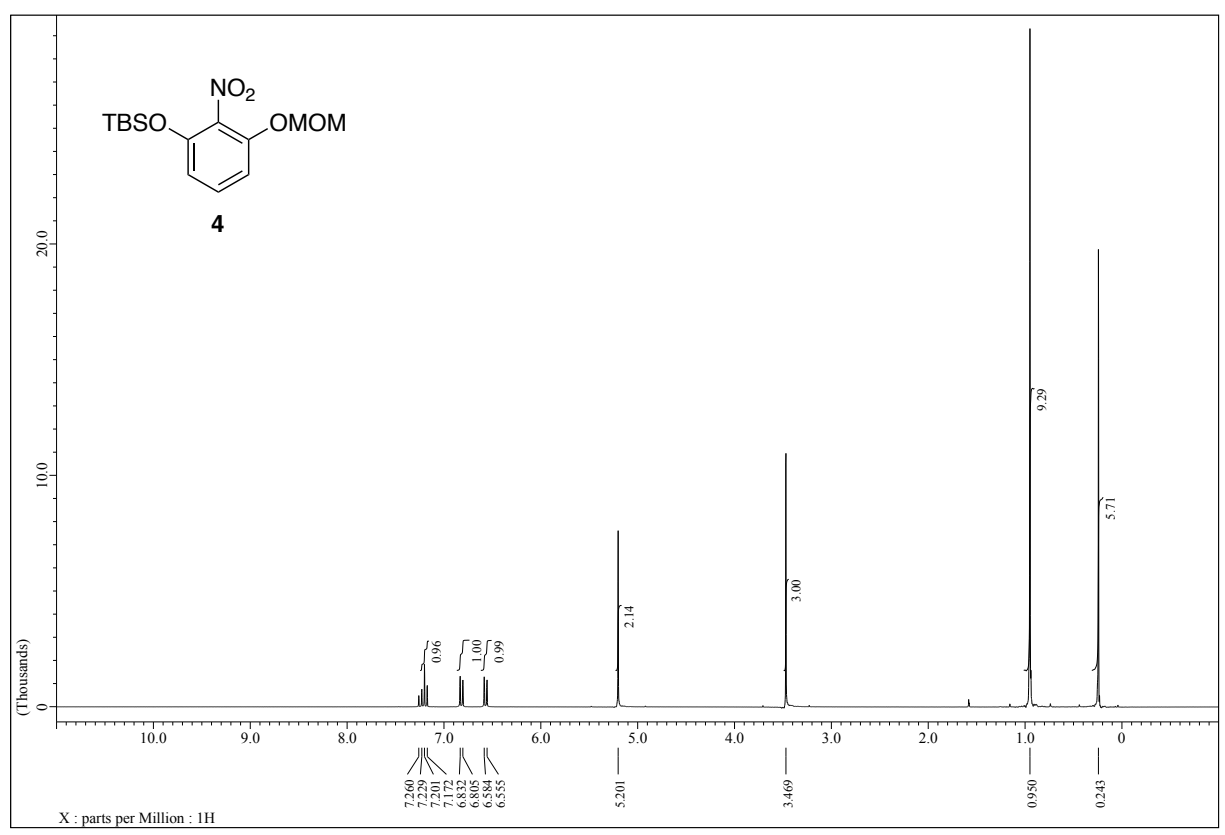

${ }^{13} \mathrm{C}$ NMR Spectrum of $4\left(75 \mathrm{MHz}, \mathrm{CDCl}_{3}\right)$

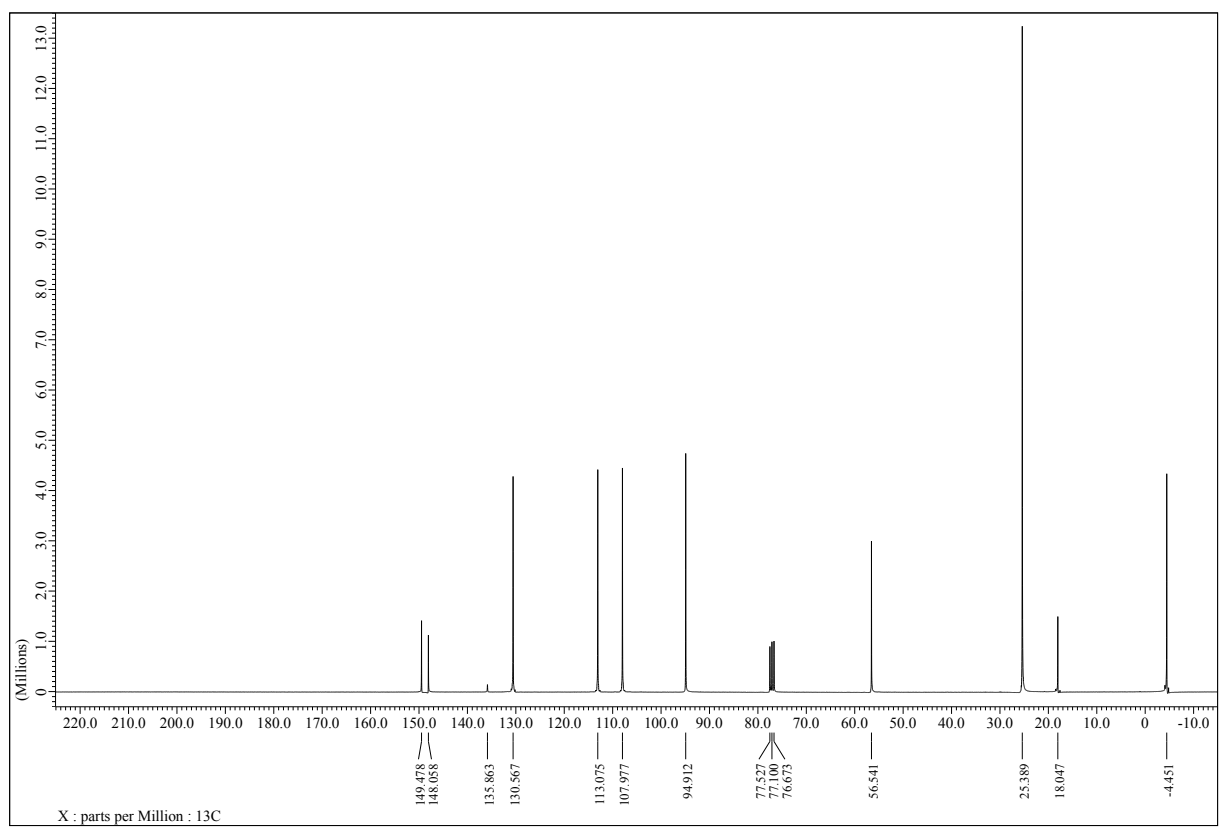


${ }^{1} \mathrm{H}$ NMR Spectrum of $5\left(300 \mathrm{MHz}, \mathrm{CDCl}_{3}\right)$

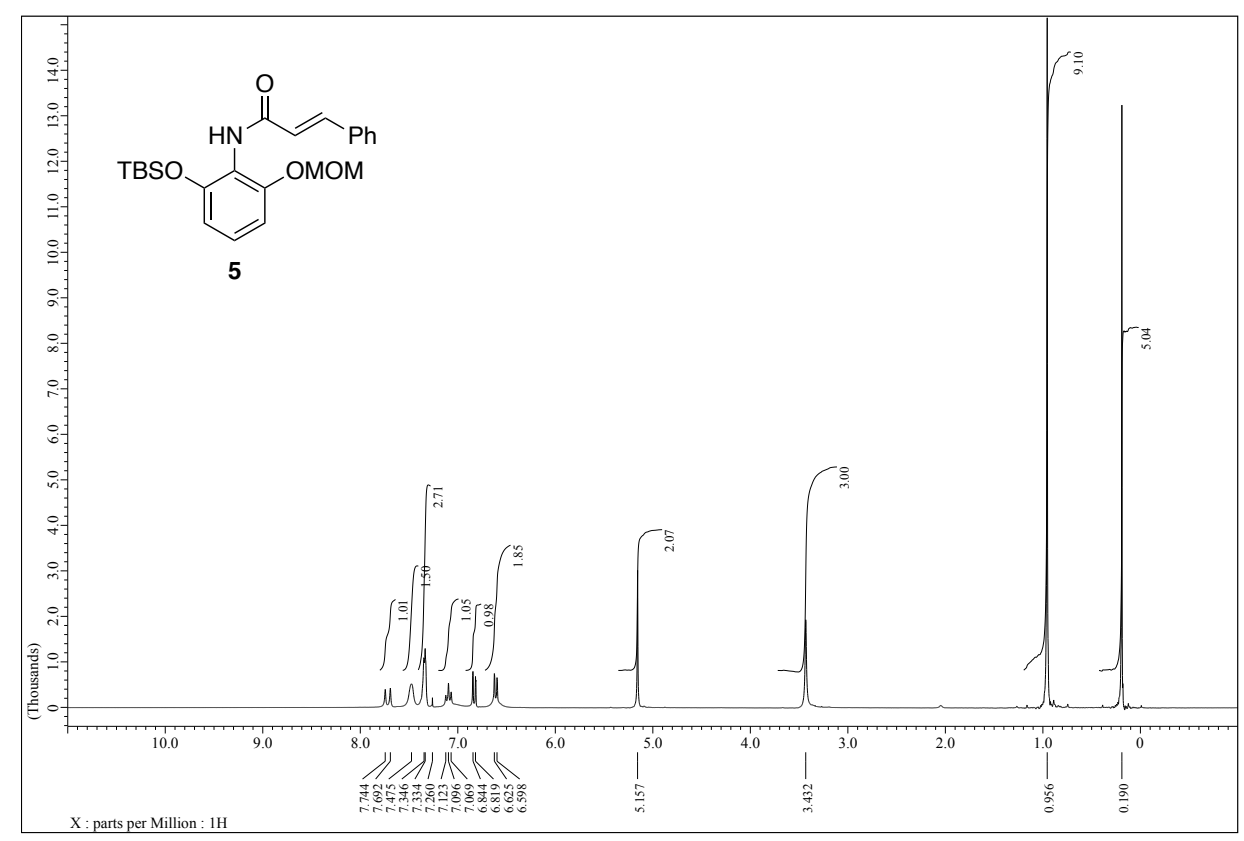

${ }^{13} \mathrm{C}$ NMR Spectrum of $5\left(75 \mathrm{MHz}, \mathrm{CDCl}_{3}\right)$

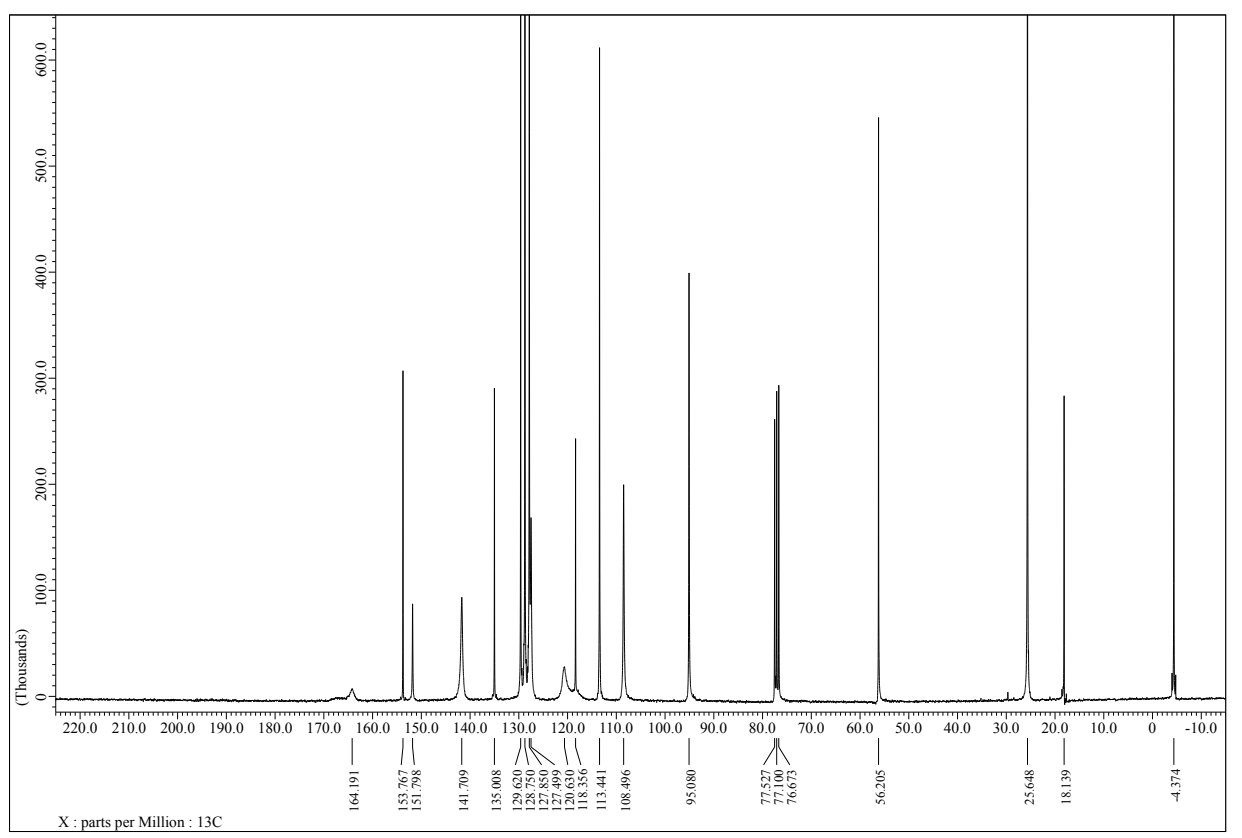


${ }^{1} \mathrm{H}$ NMR Spectrum of $6\left(300 \mathrm{MHz}, \mathrm{CDCl}_{3}\right)$

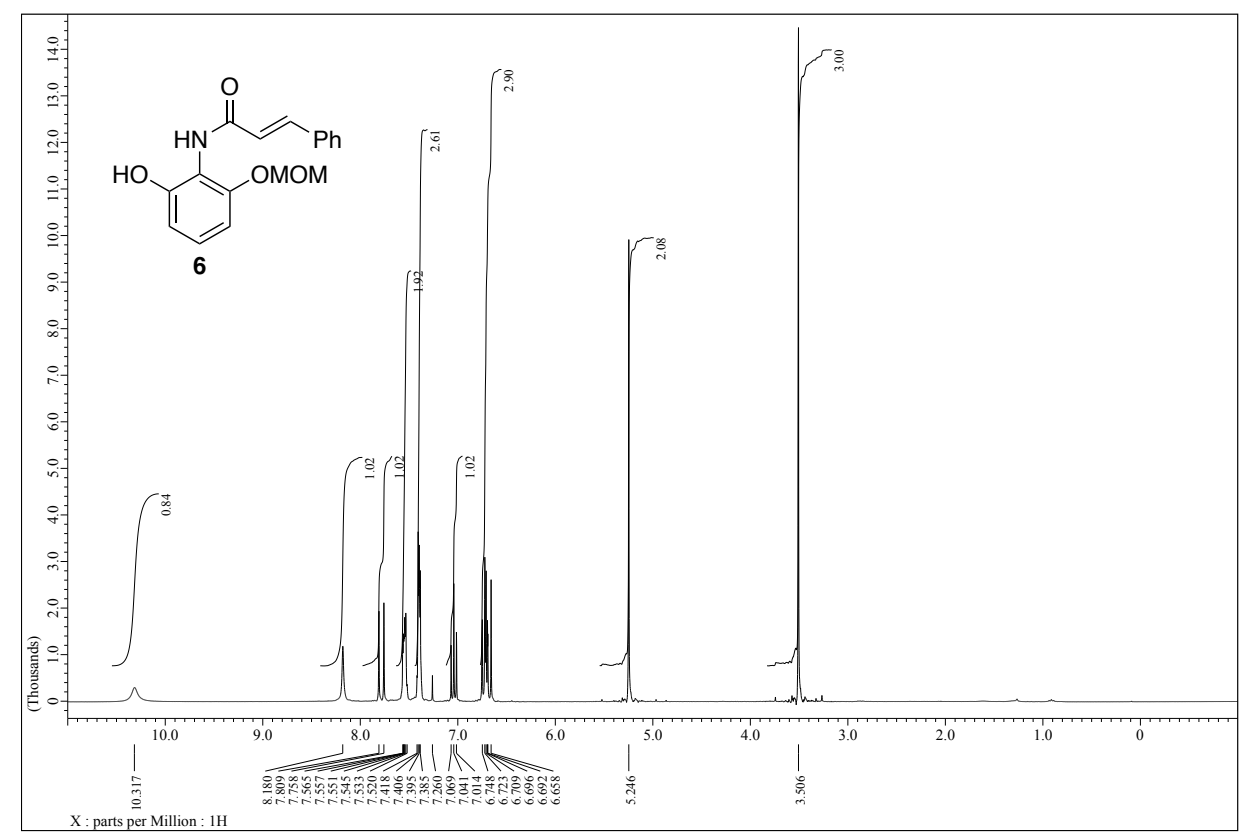

${ }^{13} \mathrm{C}$ NMR Spectrum of $6\left(75 \mathrm{MHz}, \mathrm{CDCl}_{3}\right)$

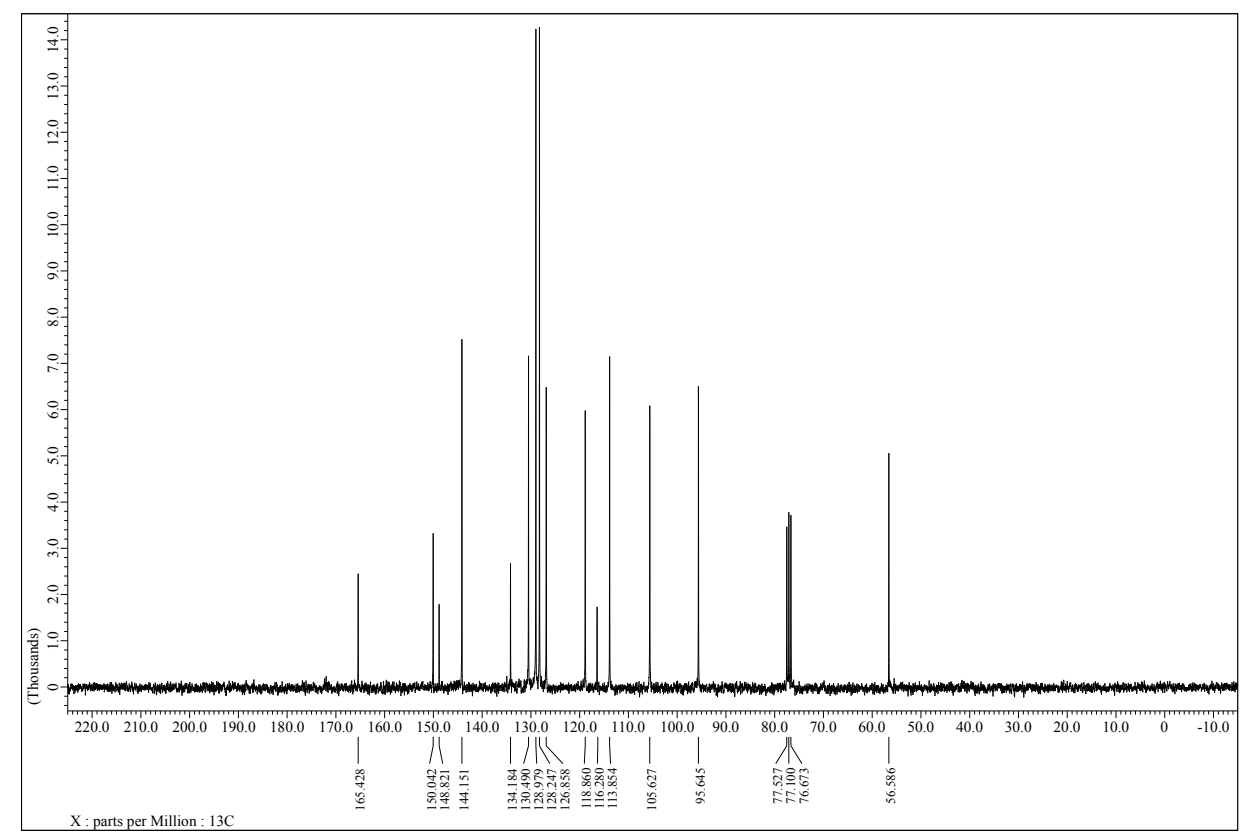


${ }^{1} \mathrm{H}$ NMR Spectrum of $7\left(300 \mathrm{MHz}, \mathrm{CDCl}_{3}\right)$

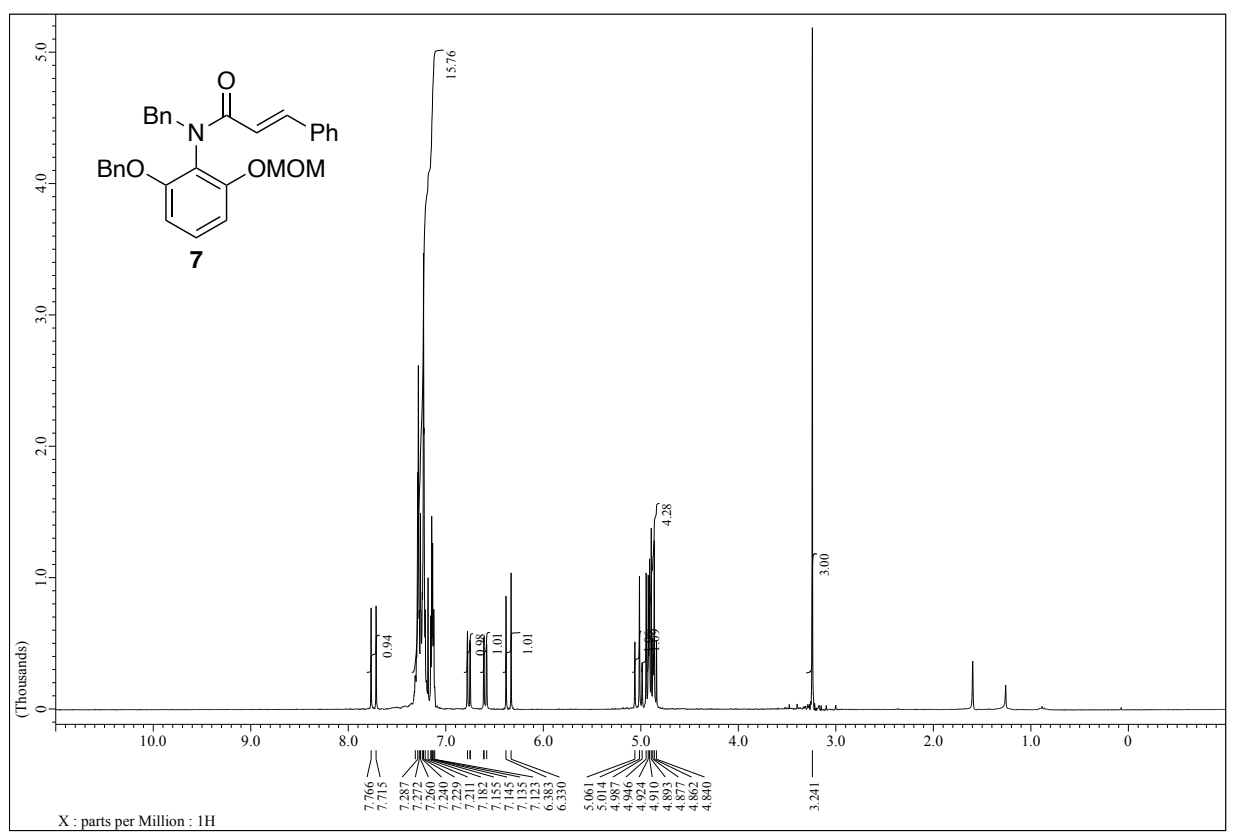

${ }^{13} \mathrm{C}$ NMR Spectrum of $7\left(75 \mathrm{MHz}, \mathrm{CDCl}_{3}\right)$

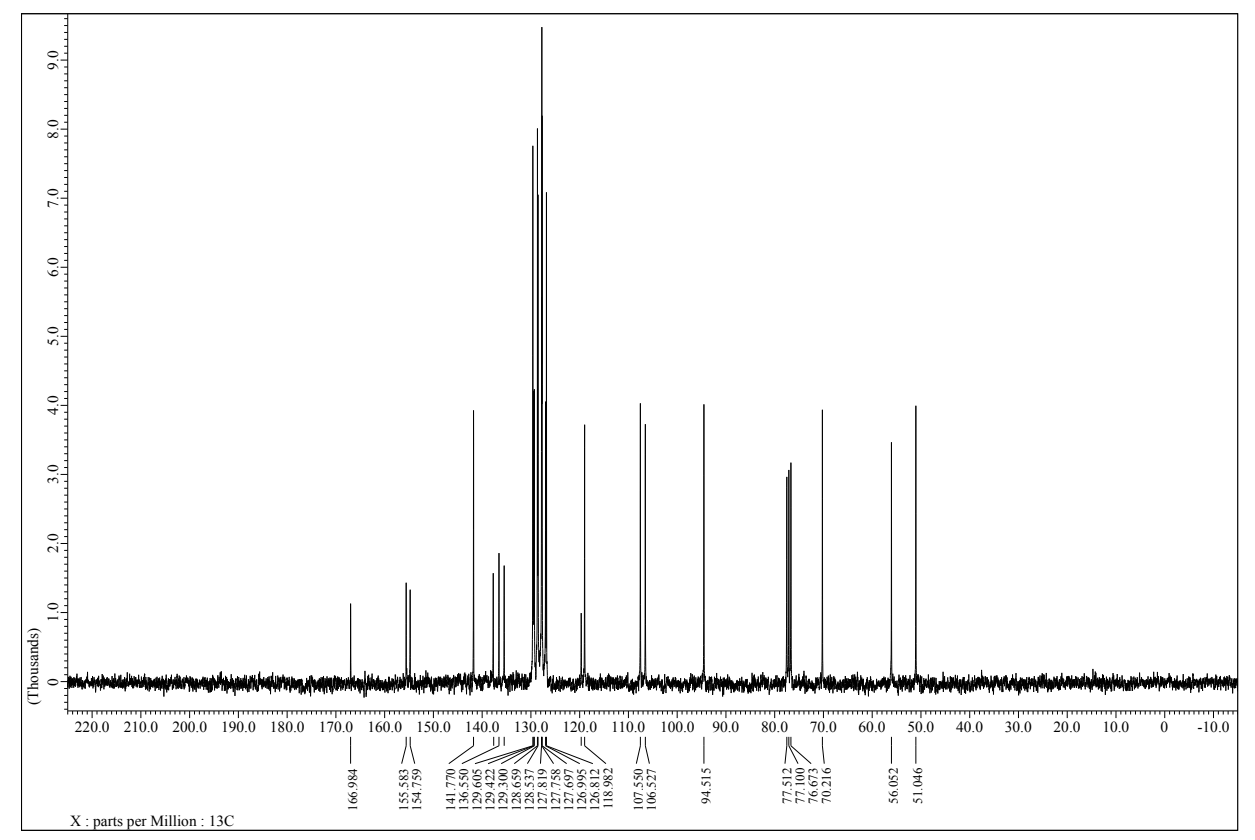


${ }^{1} \mathrm{H}$ NMR Spectrum of $\mathbf{2 a}\left(300 \mathrm{MHz}, \mathrm{CDCl}_{3}\right)$

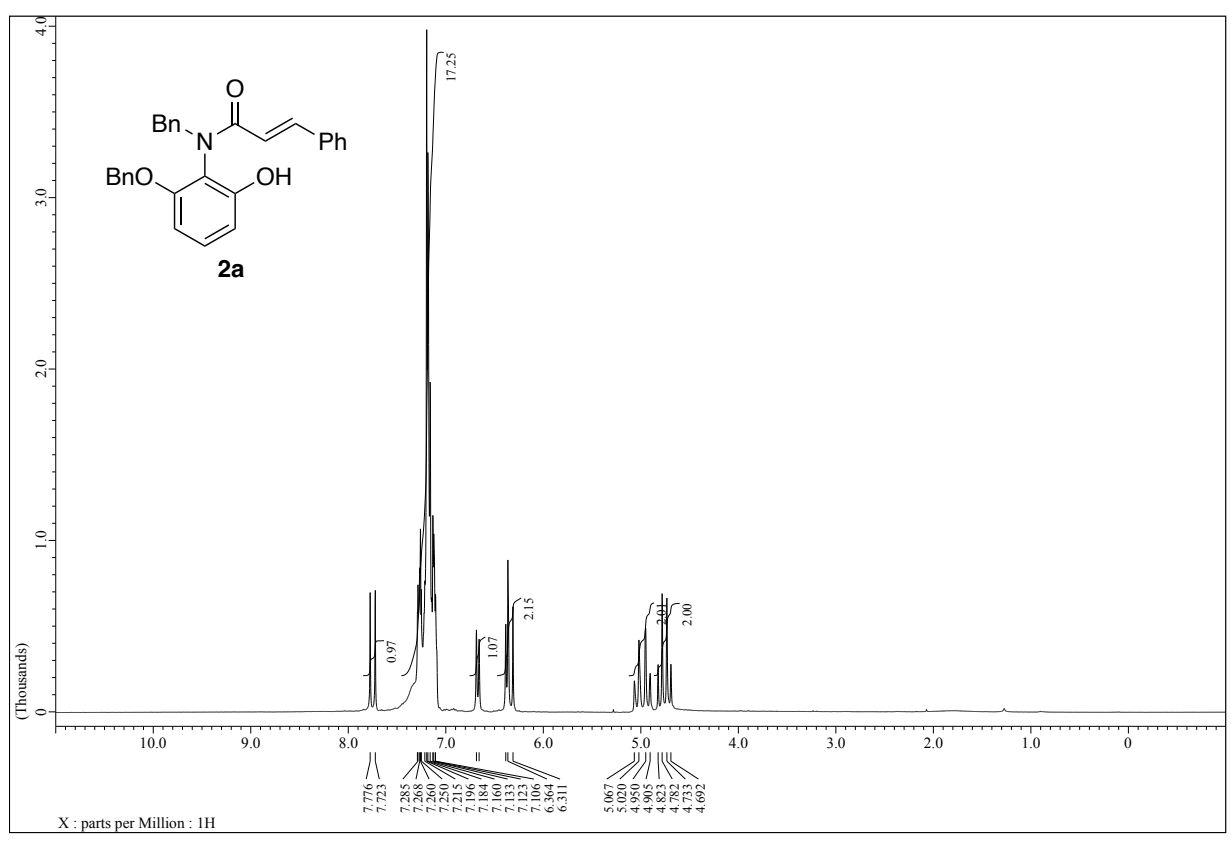

${ }^{13} \mathrm{C}$ NMR Spectrum of $\mathbf{2 a}\left(75 \mathrm{MHz}, \mathrm{CDCl}_{3}\right)$

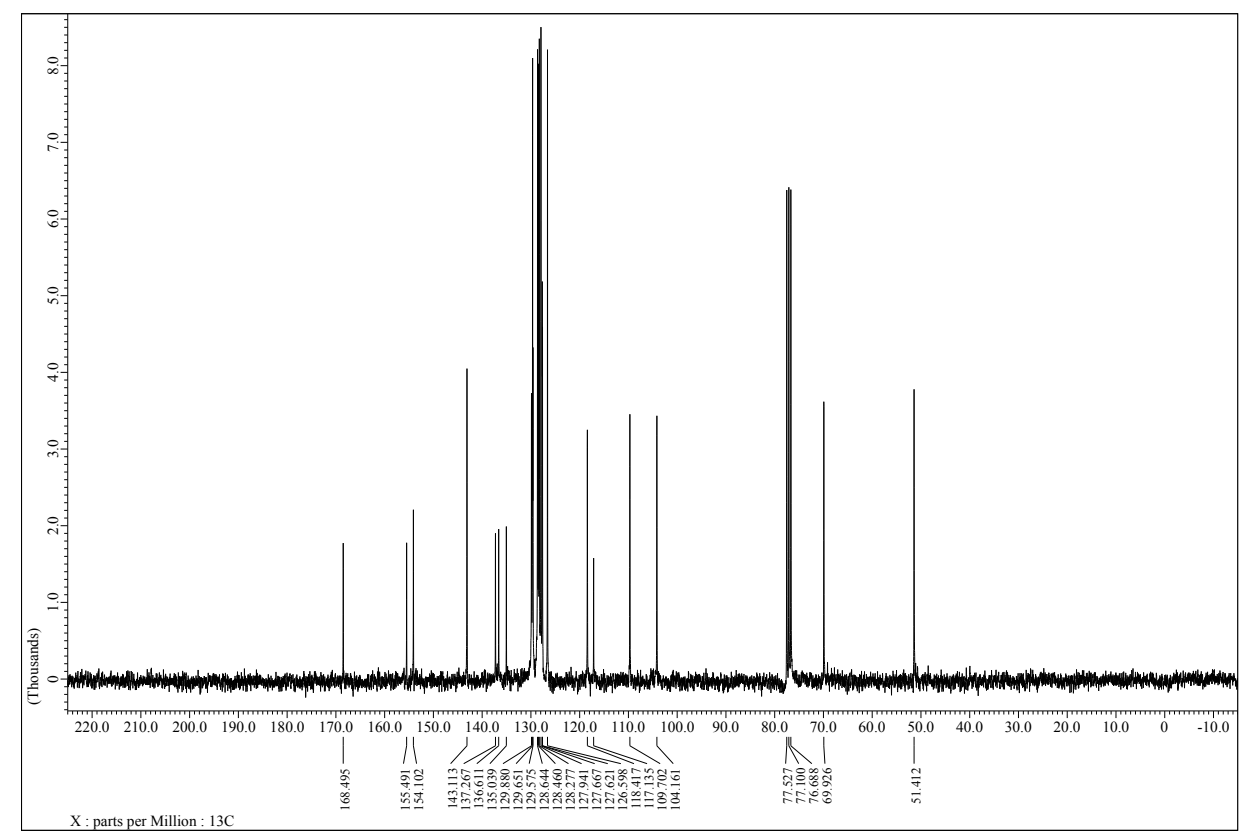

